\title{
Nursing Experience during Perioperative Period of Cervical Spinal Cord Injury
}

\author{
Caili $\mathrm{Li}^{*}$ and Hao Xu \\ Department of Orthopedics, The Second Hospital of Taizhou City, Taizhou, Zhejiang 318000, China
}

\begin{abstract}
Objective: To discuss the nursing experience during the perioperative period of cervical spinal cord injury. Method: Retrospectively analyze the clinical data of cervical spinal cord injury cases treated by our department from January to December of 2012 and apply the preoperative nursing and postoperative nursing for them. Results: The overall incidence of severe complications is $73.3 \%$ during the perioperative period, including hyponatremia of $46.7 \%$, respiratory failure of $6.7 \%$, urinary system infection of $40 \%$, pulmonary infection of $26.7 \%$, and stress ulcer of $26.7 \%$. There is 1 case died of the disease before operation but no postoperative death, and the death rate during perioperative period is $6.7 \%$. Conclusion: The rehabilitation nursing for patients with cervical spinal cord injury during perioperative period can reduce the complication and improve life quality, so it is worth being popularized.
\end{abstract}

\section{KEYWORDS}

Cervical spinal cord injury

Perioperative nursing

Spinal cord injury

\section{Introduction}

The high and middle cervical spinal cord injury caused by the cervical fracture and dislocation is a common clinic trauma with higher incidence of disability and death. The patient of this disease is in a critical condition with complicated disease and difficult nursing who often requires operative treatments. Enhancing the perioperative nursing plays an important part in improving rehabilitation and reducing the appearance of complications. Now, I will analyze the cases of cervical spinal injury collected and cured in the whole year of 2012 retrospectively.

\section{Clinical Data}

There are 15 cases in total in this group, including 12 males and 3 females; the age range is $23-74$ and the average age of them is 53 . The pathogenesis includes 10 cases of traffic accidents, 4 cases of fall accident from high place and 1 case of other causes. Among them, there are 11 cases

Copyright $\odot 2013$ Caili Li and Hao Xu

doi: $10.18686 /$ jn.v2i1.5

Received: September 8, 2013; Accepted: October 16, 2013; Published online: December 22, 2013

This is an open-access article distributed under the terms of the Creative Commons Attribution Unported License (http://creativecommons.org/ licenses/by-nc/4.0/), which permits unrestricted use, distribution, and reproduction in any medium, provided the original work is properly cited.

${ }^{*}$ Corresponding author: Department of Orthopedics, The Second Hospital of Taizhou City, Taizhou, Zhejiang 318000, China. E-mail: 786399274@qq.com of one vertebral fracture and 4 cases of two adjacent vertebral fractures. The time from injury occurrence to the admission of the department is 2 hours to 3 days and the operation is conducted after 3 days to 15 days since the admission to the department and the average days is 9 . The overall incidence of severe complications is $73.3 \%(11 / 15)$ during the perioperative period, including hyponatremia of $46.7 \%$ (7/15), respiratory failure of $6.7 \%(1 / 15)$, urinary system infection of $40 \%(6 / 15)$, pulmonary infection of $26.7 \%(4 / 15)$, and stress ulcer of $26.7 \%(4 / 15)$. There is 1 case died of the disease before operation and no postoperative death. The death rate during perioperative period is $6.7 \%(1 / 15)$.

\section{Nursing \\ 3.1. Pre-operative nursing \\ 3.1.1. Mental nursing}

Patient with cervical spinal cord injury usually lose their normal physiological functions at that moment. Due to the sudden pain, urgent illness and obvious symptoms, patients fail to have mental preparation for facing the severe injury and high death risk, so patients usually are nervous with anxiety, fear and impatience. The unsteady spirits will influence the treatment effect and conditions of prognosis. The positive and effective psychotherapy is important for the patient before operation, and the features of the disease and the importance of the surgery shall be explained to patients carefully to relieve the anxiety, so that patients have the best status for the treatment. 


\subsubsection{Vital signs and illness monitoring}

The spinal cord edema will occur after the cervical spinal cord injury and the most serious time usually occur after 48 hours of being injured. The serious edema can lead to respiratory muscle paralysis which can cause respiratory failure, so patients will suffer aggravation and even death risk before operation. At the time, the conscious state and vital signs of the patients shall be closely observed (especially the respiratory rate and respiratory rhythm shall be paid more attention to). In this group, a patient died from respiratory failure on the second day of getting cervical spinal cord injury. For patients who accept pulsedose therapy with large dosages of methylpred disolonum within 6 hours after getting cervical spinal cord injury, the abdominal sign and stool characters shall be monitored to inspect whether there is stress ulcer complication or not. The incidence of stress ulcer is $26.7 \%$ in this group. The abnormal vital signs and aggravation shall be informed to the physician for the timely treatment.

\subsubsection{Traction nursing}

Since the cervical fracture and dislocation broke the stability of cervical vertebra, cervical traction for reposition and immobilization are normally required to prevent the secondary injury and relieve the local pain caused by the nerve root compression. When the body is turned over, the head, neck and shoulder of the patient shall be kept in the same plane and the traction shall be on the axle wire to ensure effective traction. The sustained traction shall be informed to the patient and family, and if it is necessary to adjust the traction, the operation shall be conducted by the medical personnel. Both sides of the head shall be secure by 2 punch bags to prevent the double swerve.

\subsubsection{Anterior trachea over training}

The purpose of the training is to make the patient adapt to the traction of weasand and esophagus during the operation, so that the centrum can be exposed for facilitating operation. The training shall be stopped timely with the conditions of irritating cough and nausea, and the patient needs take a deep breath. If the patients fail to operate by themselves, the nurse and family shall assist.

\subsection{Post-operative nursing}

\subsubsection{Vital signs monitoring}

Electrocardiogram monitoring shall be provided persistently after operation, including monitoring of blood pressure, heart rate, breath and oxyhemoglobin saturation. Since the paralysis of intercostal muscles and diaphragm caused by the cervical cord injury may persist and there are postoperative complications, such as laryngeal edema, incision hematoma and so on, dyspnea will be easily caused, so it is necessary to monitor the respiratory rate, the shallow and deep degree of breath and tone of breath to make sure there is no suffocation, dyspnea, blood oxygen de- saturation and so on. Once the abnormal phenomenon is found, the physician shall be informed for the timely treatment, in addition, the continuous low-flow oxygeninhalation is necessary. The tracheotomy set shall be equipped at the bedside so that the tracheotomy and hematoma incision drainage can be operated once asphyxia occurs.

\subsubsection{Dietary nursing}

The patient may suffer dysphagia and foreign body sensation at the throat after operation due to the tracheoesophageal traction during anterior cervical surgery. The patient shall be guided to have much cold drink to relieve the irritation of throat within 24 to 48 hours after operation. Bland digestible semi-liquid food is suitable for the patient. For the patients who eat less and is in a critical condition, the parenteral nutrition shall be provided.

\subsubsection{Hyponatremia nursing}

Hyponatremia is one of the common complications of cervical cord injury and the incidence of hyponatremia is 46.7 $\%$ in this group. The reduction of $\mathrm{Na}^{+}$in the serum can lead to cellular edema, internal environment disturbance and exacerbate the nerve injury. The common unspecific manifestations include: poor spirit, in appetence, weakness, and fatigue; digestive system manifestations include: abdominal distension, nausea and emesis; respiratory system manifestations include: shallow breathing, cough and expectoration weakness; circulatory system manifestations include: edema of lower extremity and scrotum. The daily monitoring for the change of serum $\mathrm{Na}^{+}$and urinary $\mathrm{Na}^{+}$ is necessary to prevent the hyponatremia. When the serum $\mathrm{Na}^{+}$level is more than $130 \mathrm{mmol} / \mathrm{L}$, the sodium chloride for oral with the routine fluid replacement is enough for treatment; when the serum $\mathrm{Na}^{+}$level is less than 130 $\mathrm{mmol} / \mathrm{L}$, the intravenous infusion of $\mathrm{Na}^{+}$shall be provided, the dosage of supplemented sodium shall not be too large, the speed of sodium supplement shall be under control, in addition, what is deserved to mention is that the hypertonic saline is not suitable for the treatment because it may lead to central pontine myelinolysis with worse prognosis [1].

\subsubsection{Respiratory nursing Preventing respiratory tract infection}

The incidence of respiratory tract infection is $26.7 \%$ in this group. Due to the paralysis of respiratory muscle, decreased vital capacity, post-operative pain and so on, patients will suffer cough weakness which will influence sputum excretion, cause chronic infection and high treatment cost. The respiratory tract shall be kept fluent with timely secreta clearing, oxygen inhalation and atomization. $\mathrm{Pa}$ tients should be encouraged for deep breath and effective cough during the nursing. As for the patients who fail to cough the sputum by themselves, the sputum suction shall be offered to assist. Before sputum suction, the posture 
therapy of slapping back to excretory sputum can be given; during the treatment, facing the patient is necessary to inspect the breath state to make sure there is no asphyxia. The sputum suction must be conducted at the bacteria-free environment. The best way to prevent pulmonary infection is encouraging the patients to sit up in bed, and elevate the bed of $30-40^{\circ}$ for the patients without special taboo, which is in favor of smooth breathing and sputum excretion [2].

\section{Respiratory function training}

When patients wake up from the anesthesia after operation and their vital signs are stable, patients shall be instructed to conduct exercise of shrinking lip breathing, deep-breath exercising pulmonary function and passive breathing exercise to increase lung capacity and improve the recovery of respiratory function [3].

\section{Defecation nursing}

Incidence rate of fecal dysfunction is higher after cervical cord injury. When it happens in the acute phase, the fecal dysfunction presents astriction and urinary retention, so we can use laxatives and retention catheterization in ordinary. However, long-term retention catheterization is very easy to cause the infection of urinary system. The incidence of infection in this group is $40 \%$ caused by the longterm retention catheterization. It is essential to encourage patients to drink appropriate water and if necessary, give them furacilinum of $0.02 \%$ and conduct bladder washing of $500 \mathrm{~mL}$ once to twice every day to prevent infection of urinary tract. After the recovery of bladder functions, the retention catheterization can be stopped as early as possible, which can reduce the incidence of urinary infection effectively [3].

\subsubsection{Motor function nursing}

(1) Limbs functional training: assist patients in doing extensor and plantar flexion of ankles, and extensor, abduction and snatches of upper limbs on the day of the operation; make patients have active and passive functional exercises of every articulation of limbs and the muscular atrophy on the first day after the operation; train patients' muscles and joint stretch in a week after the operation, including trainings on hamstring stretch, adductor stretch and Achilles tendon stretch. The training should be conducted three to five times every day, each joint should be exercised twenty to thirty once time, and then gradually increases times and time. (2) Sitting position training: lift the head of the sick bed to make patients turn their semisitting positions to the lateral positions. When conducting the trainings, the family cannot leave away from the patient to prevent the second injury from falling down from the bed. (3) Tilting table exercise: lower limbs of the patient should be equipped with braces with the help of the walker and assistance from families, time of training can be increased gradually (4) Transfer training: this training includes the transfer from lying position to sitting position, transfer from bed to bedside and transfer from bedside to wheelchair and so on. (5) Wheelchair operation training: Tell main points and method of using wheelchairs to the patient. The sitting position of the wheelchair means the body center of gravity should be in the front or at the back of ischial tuberosity. When wheelchair time is longer than 30 minutes, the buttock should be lifted to avoid pressure sores which may happen in the position of ischial tuberosity [4].

\subsection{Rehabilitation guidance}

The purpose of rehabilitation guidance lies in recovering the residual functions of the patient furthest, improving the quality life of the patient and reducing the burdens on the families and society. When the vital signs of patients are stable, a series of rehabilitation interventions conforming to the actual conditions of the patient should be provided to improve the recovery of function. Principles of nursing are starting as soon as possible, perseverance, priority to the easy, step by step and combination of active and passive. In the early process of rehabilitation nursing, we should explain relevant medical knowledge to the patient and families, introduce methods and significances of relevant treatment, nursing and rehabilitation of spinal cord injuries, and achieve the cooperation of the patient which can develop the patient's subjective initiative. Guarantee the patients can obtain long-term, systematic and rational training in the family after leaving the hospital to achieve the final objective of rehabilitation, which benefits the patient to keep a long-term ability of independent living and return to the society [5].

\section{Results}

Limb dysfunction or body paralysis appears usually after the cervical spinal cord injury, which makes the patient lose all or part of life self-care ability. Combining with serious complications of pressure sore and pneumonia, it leads to the fatal physical and mental trauma even the lifetime disability of the patient. The patient suffering from the cervical spinal cord injury has got different level limbs disability, and sense dysfunction except the pain of position in the injury. Secreta staying in the lung will cause the hypostatic pneumonia because of the weakened protective reduction, such as cough and sneezing; the retention of urine and the infection of urinary system may be caused by kinds of reasons; pressure ulcer may be caused because of long compressed local issues, malnutrition and lower temperature; the damage of sympathetic nerve causes the hyperpyrexia and hypothermia, which may make patients produce a strong psychological wave motion. In the acute phase, the patients always express their desperation and concerns and they may even have suicidal thoughts. Aiming at these problems mentioned above, we should provide corresponding nursing measures education and rehabilitation nursing so as to eliminate the anxiety of patients, help 
them regain their confidence and make them cooperate with the functional trainings actively.

The data of this group shows that the incidence of severe complications is $73.3 \%$ during perioperative period, including hyponatremia of $46.7 \%$, respiratory failure of $6.7 \%$, urinary system infection of $40 \%$, pulmonary infection of $26.7 \%$, and stress ulcer of $26.7 \%$. There is 1 case died of the disease before operation and no postoperative death, and the death rate during perioperative period is $6.7 \%$. With our carefully nursing, closer observations, timely treatment and safe measures, there is no complication and the patient is satisfied with the recover condition, short course of treatment and treatment effect.

\section{Conclusion}

In conclusion, the complications of cervical spinal cord injury are more and serious. Most patients die from these complications, so the nursing is important. Increasing the nursing input to respiratory system can decrease or avoid these complications and reduce unnecessary medical input. Surviving from the perioperative period has an important significance to increasing patients' high survival rate and life quality, and making them return to society.

\section{Conflicts of interest}

These authors have no conflicts of interest to declare.

\section{Authors' contributions}

These authors contributed equally to this work.

\section{References}

1. Du X, Song L, Xu J. Clinical features of central pontine myelinolysis and its prognosis of recovery. Chinese Journal of Rehabilitation Theory and Practice. 2011;14(9):852-855.

2. Liu $X$, Jin $X$, Zhao G. Sputum drainage nursing of patients with lung infection. Chinese Journal of Nursing. 2007;33(12):697.

3. Chen Y. The analysis of reasons and nursing countermeasures induced urinary tract infect by the patients with retention catheterization. Attend to Practice and Research. 2007;4(11):18-19.

4. Jiang $\mathrm{H}$. Early rehabilitation intervention in patients with spinal cord injury. Modern Practical Medicine. 2009;21(11):1260-1261

5. Wang G, Ge X. Perioperative nursing experience of patients with cervical spinal injury. Traditional Chinese Bone-Setting. 2010;22(10):78-79. 